\title{
Water entry of spheres at various contact angles
}

\author{
Nathan B. Speirs ${ }^{1}$, Mohammad Mansoor ${ }^{1}$, Jesse Belden ${ }^{2}$ and Tadd \\ T. $\operatorname{Truscott}^{1} \dagger$ \\ ${ }^{1}$ Department of Mechanical and Aerospace Engineering, Utah State University, Logan, UT \\ 84322, USA \\ ${ }^{2}$ Naval Undersea Warfare Center Division Newport, 1176 Howell Street, Newport, Rhode \\ Island 02841, USA.
}

(Received xx; revised xx; accepted xx)

It is well known that the water entry of a sphere causes cavity formation above a critical impact velocity as a function of the solid-liquid contact angle (Duez et al. 2007). Using a rough sphere with a contact angle of $120^{\circ}$, Aristoff \& Bush (2009) showed that there are four different cavity shapes dependent on the Bond and Weber numbers (i.e., quasistatic, shallow, deep and surface). We experimentally alter the Bond number, Weber number and contact angle of smooth spheres and find two key additions to the literature: 1) Cavity shape also depends on the contact angle; 2) the absence of a splash crown at low Weber number results in cavity formation below the predicted critical velocity. In addition, we find an alternate scaling for the Bond and Weber numbers that predicts cavity shapes for various impacting bodies (e.g., spheres, multi-droplet streams and jets) on the same regime diagram, thus, merging the often separated studies of solid-liquid and liquid-liquid impact in the literature.

\section{Introduction}

At the turn of the millennium a resurgence of interest occurred in water entry studies. During this time, two foundational papers on cavity formation and cavity dynamics were published, which describe the regimes into which all other water entry studies fall. The first was written by Duez et al. (2007) who investigated when impacting spheres form air cavities, as shown in figure 1b-e, and when they do not, as shown in figure 1a. The second paper, written by Aristoff \& Bush (2009), came along a couple of years later discussing the four different shapes or types of cavities that form once the appropriate conditions are met for cavity formation (shown in figure 1b-e). We will now examine each of these papers in turn, discussing their findings, an unaddressed discrepancy between the two, and how this paper expands our understanding of the conditions in which these five water entry regimes occur.

Duez et al. (2007) found that cavity formation of smooth spheres occurs above a critical velocity $U_{c r}$ that is a function of the advancing static contact angle $\theta$. Hydrophilic spheres $\left(\theta<90^{\circ}\right)$ form cavities above $U_{c r} \approx 7.2 \mathrm{~m} / \mathrm{s}$ in water. The critical velocity decreases for hydrophobic spheres $\left(\theta>90^{\circ}\right)$ going to zero as $\theta$ goes to $180^{\circ}$. They explain this finding by discussing the contact-line stability of the thin, upward-moving film or splash that forms around the circumference of the sphere upon impact. When the splash adheres to the sphere (below the critical velocity) no cavity forms. When it separates from the sphere, air gets pulled behind the sphere and a cavity forms.

$\dagger$ Email address for correspondence: taddtruscott@gmail.com 


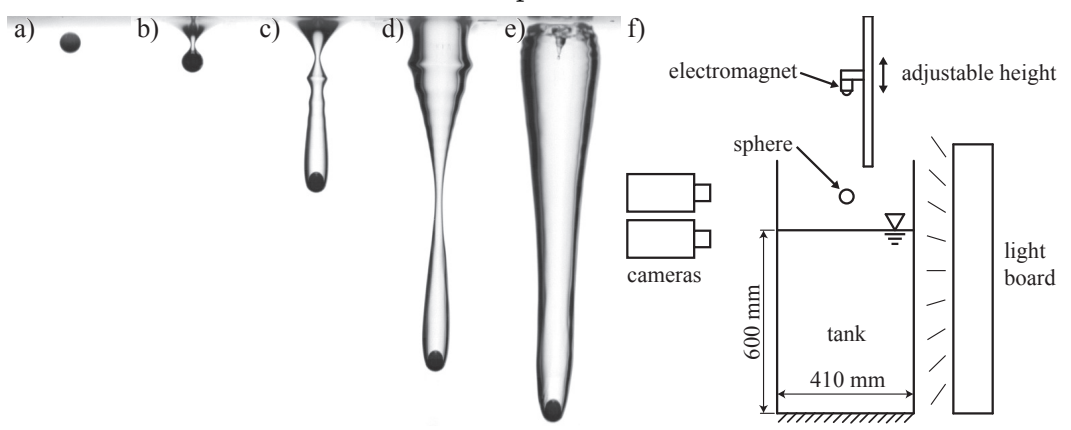

FiguRE 1. A $2 \mathrm{~mm}$ diameter sphere impacts the water surface creating various cavity types. In a) the wetting angle is $\theta=101.0^{\circ}$ and the sphere impacts at $4.43 \mathrm{~m} / \mathrm{s}$ without forming a cavity. In b) through e) the sphere has a wetting angle of $\theta=141.1^{\circ}$ and impacts with velocities $0.24 \mathrm{~m} / \mathrm{s}, 1.40 \mathrm{~m} / \mathrm{s}, 2.80 \mathrm{~m} / \mathrm{s}$, and $4.43 \mathrm{~m} / \mathrm{s}$ forming quasi-static, shallow, deep, and surface seal cavities, respectively. See supplementary movies 1-5. f) The basic experimental setup is shown with two high-speed camera viewing the impact events above and below the free surface.

Aristoff \& Bush (2009) studied the water entry of spheres with one contact angle, $\theta=120 \pm 5^{\circ}$, and various impact velocities and sphere diameters. Their expansive data set found cavities forming at all impact velocities with four distinct shapes defined by their collapse or pinch-off location, each of which occurs at a specific location on a Bond-Weber plot. At the lowest Weber number, $W e$, they describe quasi-static seal, in which pinch-off occurs on or very near the sphere surface (figure 1b). At higher $W e$ both shallow and deep seal are seen. Shallow seal occurs at lower Bond number, $B o$, where surface tension dominates and the pinch-off depth is on the order of the capillary length (figure 1c). Deep seal occurs at higher $B o$, where gravitational forces dominate and pinch-off occurs about half way between the pool surface and the sphere (figure 1d). Surface seal occurs at the highest $W e$, wherein the splash created upon impact collapses inward due to air pressure and surface tension (Marston et al. 2016) sealing at the pool surface (figure 1e).

According to Duez et al. (2007), at the contact angle used by Aristoff \& Bush (2009) $\left(\theta=120^{\circ}\right)$, cavities should not form below $U_{c r}=2.14 \mathrm{~m} / \mathrm{s}$. Yet Aristoff \& Bush report quasi-static, shallow and deep seal cavities at velocities below this value. We can explain this discrepancy by the high surface roughness of the coating used by Aristoff \& Bush, Cytonix WX2100, which, in our own testing we have found to have a large ten-pointmean roughness of $R_{z}=50.2 \pm 21.4 \mu \mathrm{m}$. Zhao et al. (2014) found that $U_{c r}$ is also a function of the sphere roughness $R_{z}$, with increasing roughness leading to lower values of $U_{c r}$. Cavities should always form at the above value of $R_{z}$ and hence the cavity formation described in work of Aristoff \& Bush (2009) is due to both the high contact angle and high roughness.

Since these foundational works, several other important studies have come forth. Important topics include: the water entry of spinning spheres (Truscott \& Techet 2009a,b), the effect of sphere density (Aristoff et al. 2010), the occurrence of multiple pinch-off events (Mansoor et al. 2014), the buckling instability in the crown (Marston et al. 2016), the effects of deformability (Hurd et al. 2017), the unsteady forces during entry (Truscott et al. 2012), and many more described in the annual review by Truscott et al. (2014).

Although these works have contributed much to our understanding of water entry, we will return our focus to the foundational works discussed above and examine how the cavity formation regimes respond to experimentally varying the wetting angle $\theta$, the sphere diameter $d$ and the impact velocity $U_{o}$ for smooth spheres. We will explain the 


$\begin{array}{lll}\text { Coating } & \theta & R_{z}(\mu \mathrm{m}) \\ \text { Clean Steel } & 86.1 \pm 2.2^{\circ} & 0.6 \pm 0.3 \\ \text { Turtle Wax Super Hard Shell } & 101.0 \pm 4.7^{\circ} & 0.9 \pm 0.2 \\ \text { Cytonix WX2100 } & 116.6 \pm 7.0^{\circ} & 50.2 \pm 21.4 \\ \text { Glaco Mirror Coat Zero } & 141.1 \pm 3.8^{\circ} & 1.0 \pm 0.5\end{array}$

TABLE 1. List of coatings with their advancing static contact angle $\theta$ and ten point mean roughness $R_{z}$, with the mean and $95 \%$ confidence window reported.

physics using the Bond, Weber, and Froude numbers, which we define as $B o=\rho g d^{2} / \sigma$, $W e=\rho U_{o}^{2} d / \sigma$ and $F r=U_{o}^{2} / g d$, respectively, where $\rho$ is the liquid density, $g$ is the acceleration of gravity and $\sigma$ is the surface tension. We use the diameter $d$ instead of the sphere radius as the appropriate length scale in defining the above dimensionless numbers as it results in transitional behaviors around a value of one. In $\S 4$ we will also examine an alternate method of defining the Bond, Weber, and Froude numbers that allows us to predict the cavity types for various impacting bodies on the same regime diagram.

\section{Experimental setup and description}

Figure 1f shows the experimental setup used in this study. Various diameter stainless steel spheres $\left(d_{s}=1\right.$ to $\left.18 \mathrm{~mm}\right)$ of specific gravity 7.83 are dropped from an electromagnet onto a tank of water. Two high-speed cameras record the impact on the free-surface, imaging at 2,500 frames per second both above and below the surface from the side. The height of the electromagnet controls the impact velocity of the spheres $U_{o}$, which is varied from 0.24 to $10.39 \mathrm{~m} / \mathrm{s}$. To vary the advancing static contact angle $\theta$ three different coatings (or the lack thereof) are used: clean steel, Turtle Wax Super Hard Shell car wax, and Glaco Mirror Coat Zero. Values of $\theta$ and the ten point mean roughness $R_{z}$ are shown in table 1. Roughness measurements are obtained using a profilometer. The spheres are prepared by first washing with soap and water and then rinsing with ethyl alcohol. Coatings are then applied and allowed to dry before testing. After each test the spheres are dried and then recoated to ensure consistent surface properties.

From the high-speed videos we determine whether or not a cavity forms. If a cavity does form, the cavity type is determined using the definitions described by Aristoff \& Bush (2009). Measurements are also taken from the videos to find cavity depths, diameters, and the time to pinch-off. The pinch-off depth $h_{p}$ is defined as the distance from the undisturbed free surface to the location where the cavity walls or splash pinch together or collapse (positive downward). The depth of the bottom of the sphere at the time of pinch-off, $h_{b}$, is also measured from the undisturbed free surface. The pinch-off time $t_{p}$ is defined as the time from impact to the pinch-off event. In cases where cavities do not form, we define the pinch-off time $t_{p}$ as the time when the splash closes on the top of the sphere and the pinch-off depth $h_{p}$ as the location of the top of the sphere at this time. The cavity diameter is measured at discrete depths and times and the maximum cavity diameter over time is found at discrete depths, yielding $d_{c, \max }(z)$. The average of these maximum diameters over all depths defines $d_{c}$.

\section{Cavity formation and types}

Multiple sphere diameters were tested over a large range of impact velocities for each contact angle. From these data we produce $B o-W e$ plots similar to Aristoff \& Bush 
(2009) to examine how the cavity regimes change with the contact angle (figure 2). The regime diagram for $\theta=86.1^{\circ}$ is shown in figure 2a. Duez et al. (2007) predict the critical velocity for cavity formation for a hydrophilic sphere to be $U_{c r}=0.1 \sigma / \mu=7.2 \mathrm{~m} / \mathrm{s}$, which is represented by the dashed line in figure 2a. Surface seal cavities occur above this dashed line (as shown in figure 1e) and no cavity formation is observed just below it (figure 1a). Once $W e$ has decreased below about 240, cavities start to form again, which is unexpected in light of the work of Duez et al. (2007). Below $W e \approx 240$ spheres always form quasi-static seal cavities.

Looking at the regime diagram for $\theta=101.0^{\circ}$ (figure $2 \mathrm{~b}$ ) we see a similar trend to $\theta=86.1^{\circ}$ with a few differences. The critical velocity for cavity formation as predicted by Duez et al. has decreased due to the increased contact angle and is defined by $U_{c r}=$ $\frac{7}{270} \frac{\sigma}{\mu}(\pi-\theta)^{3}=4.89 \mathrm{~m} / \mathrm{s}$. The critical $W e$ below which cavities form again does not changed with the increase in $\theta$. Below $W e \approx 240$ mostly quasi-static seal cavities form with the exception of a few shallow seal cavities at low $B o$, for which the volume of air entrained with the sphere is small, but approximately equal to the volume of the sphere (this is consistent with the cut-off defined by Aristoff \& Bush (2009)).

To understand why cavities form below a $W e \approx 240$ we look at what happens as the velocity or $W e$ increases for a given sphere diameter or Bo. Figure 3a shows the impact of a $10 \mathrm{~mm}$ diameter sphere at various $W e$ when the sphere is approximately half submerged. At the lowest $W e$ a short, thick rim forms around the edge of the sphere. This rim does not have enough upward velocity to climb up the surface of the sphere and meet itself at the pole to prevent cavity formation (Duez et al. 2007). Hence, as the sphere descends, the free-surface is pulled down with the sphere and a quasi-static seal cavity forms in its wake (figure $3 \mathrm{~b} \& \mathrm{c}, W e=7.9$ ). As $W e$ increases the rim thins and grows taller due to its increasing upward velocity, which allows it to begin to climb the surface of the sphere as seen in figure $3 \mathrm{a}$ at $W e=109$. The upward velocity and adherence of the rim to the sphere directly competes with the rate of sphere submergence. This causes the water to move up and around the top of the sphere faster as it descends below the original free-surface plane, resulting in less air entrainment and the formation of increasingly smaller cavities. Hence, the pinch-off time $t_{p}$, depth of the sphere at pinchoff $h_{b}$, and the pinch-off depth $h_{p}$, all decrease with increasing $W e$ which can be seen qualitatively in figure 3 and quantitatively in figure 4 . Once $W e>\approx 240$ (dotted line) the rim has formed into a splash which has enough upward velocity to reach the top of the sphere by the time the top of the sphere has descended to the level of the undisturbed free-surface $\left(h_{p}=0\right.$ and $h_{b}=1$, figure $\left.4 \mathrm{~b} \& \mathrm{c}\right)$, as seen at $W e=269$ in figure $3 \mathrm{~b} \&$ c. Hence, the formation of the splash (which adheres to the sphere) suppresses cavity formation and we will call the dotted lines in figures $2 \mathrm{a} \& \mathrm{~b}$ and 3 the splash formation line.

As $W e$ increases above the splash formation line the splash climbs up the sphere surface faster causing $h_{p}$ to gradually rise above the free-surface (figure $4 \mathrm{c}$ ), and decreasing both $t_{p}$ (figure $4 \mathrm{a}$ ) and $h_{b}$ (figure $4 \mathrm{~b}$ ). Around $U_{c r}$, a small asymmetric cavity forms with the splash quickly doming over the top (figure $3, W e=2696$ ). Once $U_{c r}$ is reached, the splash has enough velocity to separate from the sphere and a large surface seal cavity forms (figure 3, $W e=8081$ ). Hence, we see that cavity formation or suppression is governed by the formation and separation of the splash. Also, we find that cavity formation occurs in two regimes: at low enough $W e$ that a splash does not form, and at high enough impact velocity that the splash separates from the sphere. The boundaries of the no cavity regime are defined by splash formation and what we will call Duez cavity formation (dotted and dashed lines in figure $2 \mathrm{a} \& \mathrm{~b}$ and 3 , respectively).

Surface seal is caused by the collapse of the splash crown. In the ideal cases typically 
a)

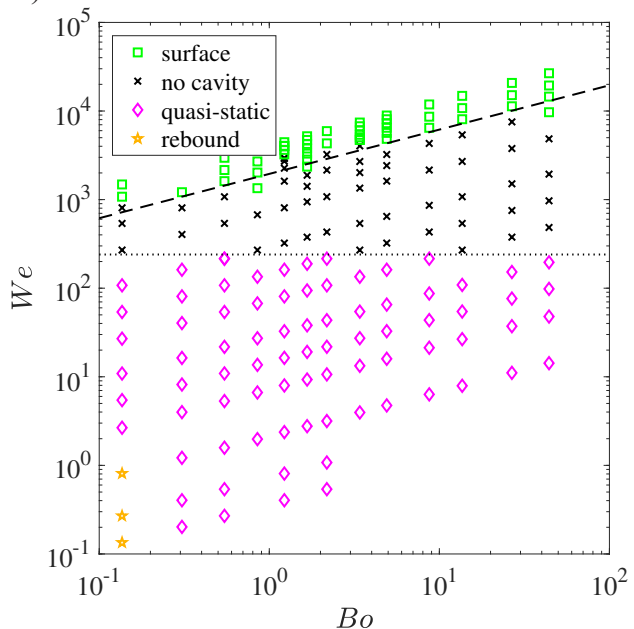

c)

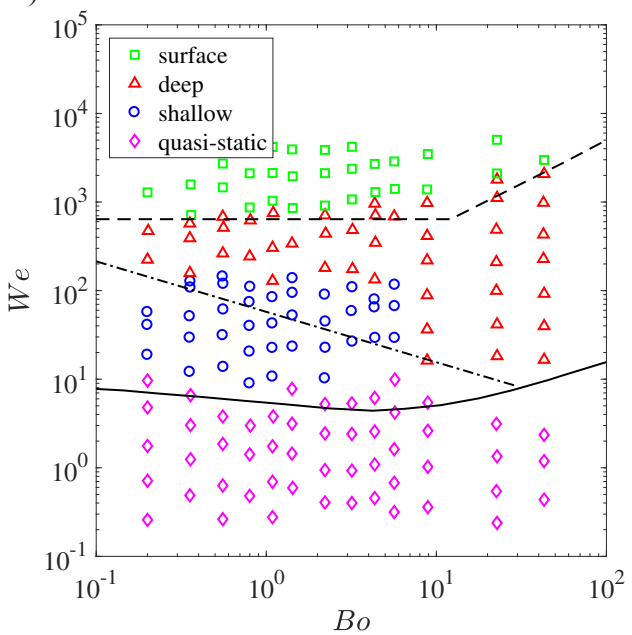

b)

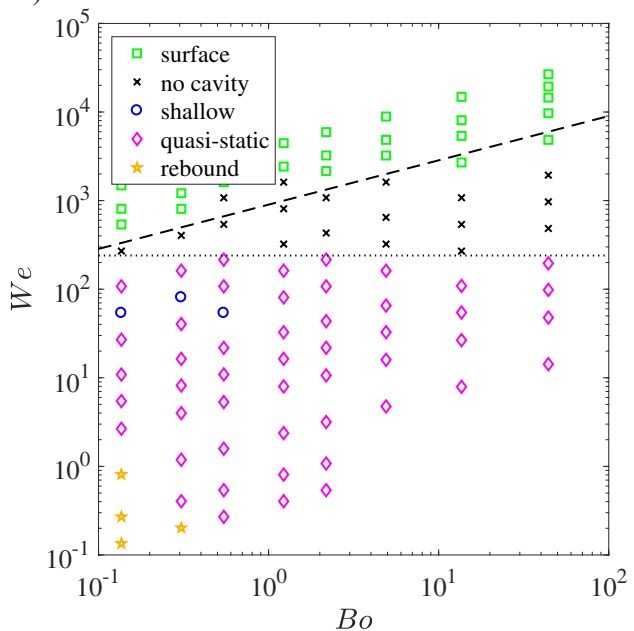

d)

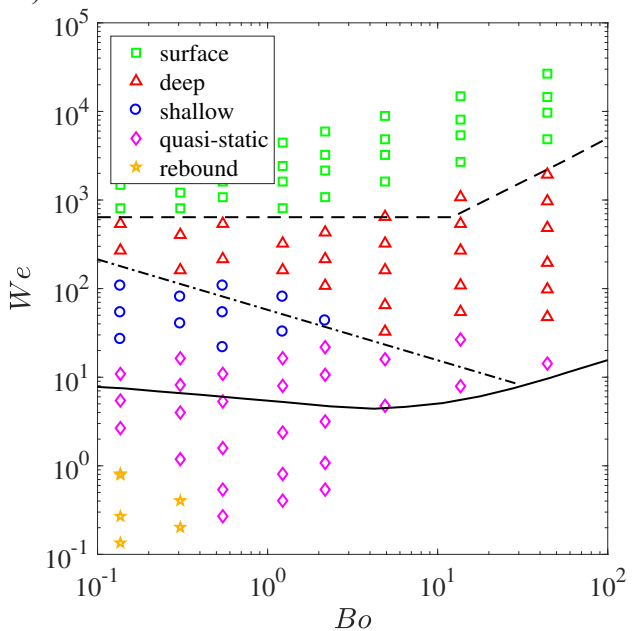

Figure 2. The cavity regimes for various contact angles are shown for a) $\theta=86.1^{\circ}$, b) $\theta=101.1^{\circ}$, c) $\theta=120^{\circ}$ and $R_{z}=50 \mu \mathrm{m}$ (remade from Aristoff \& Bush (2009)) and d) $\theta=141.1^{\circ}$. All of the spheres are smooth according to Zhao et al. (2014) except c) (table 1). The dotted and dashed lines in a) and b) represent splash formation and Duez cavity formation, respectively. The regime separation lines in c) and d) are not the same as in a) and b). Instead they come from the predictions of Aristoff \& Bush (2009). Pictures of the different regimes can be seen in figure 1a-e.

depicted in the literature, the collapse of the crown, also know as dome over, causes a complete seal between the air in the cavity and the atmosphere (see supplementary movie 5 ). Figure 5 shows an event in which a complete seal does not occur during dome over. At $t=6.4 \mathrm{~ms}$ the splash crown has domed over and the air cavity behind the sphere begins to pull away from the free surface of the pool. At $t=8.0-9.6 \mathrm{~ms}$ air continues to enter the cavity as evidenced by the small conical structure that forms and connects the top of the cavity to the surface of the pool. The conical structure then collapses radially slightly below the surface providing a complete seal $(t=11.2 \mathrm{~ms})$. These partial surface seal events could be caused by an asymmetric dome over of the splash crown, that leaves a small hole or by the formation of the thin-filmed bags observed by Marston et al. 


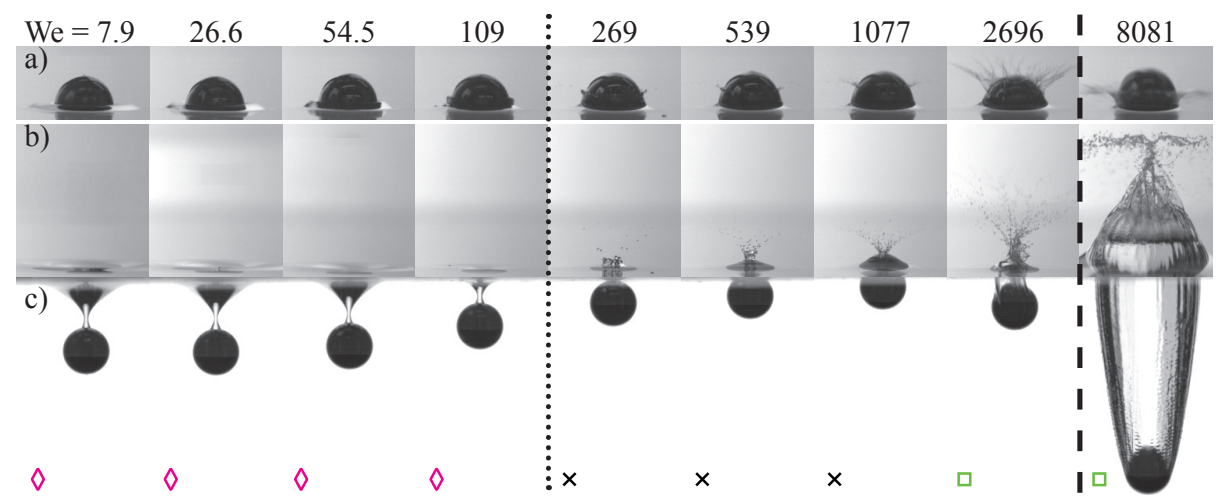

FiguRE 3. The development of the splash and progression of cavity regimes are shown for increasing $W e$, for $d=10 \mathrm{~mm}$ and $\theta=101.1^{\circ}$. Each column shows different times and/or views of the same impact event. In a) we show the development of the rim or splash when the sphere is approximately half submerged. In b) and c) we show the frame just prior to pinch-off or closure of the splash above the sphere as viewed from above and below the pool surface, respectively. The dotted and dashed lines represent splash formation and Duez cavity formation, respectively, and the symbols indicated the cavity type, which are all shown in figure $2 \mathrm{~b}$.
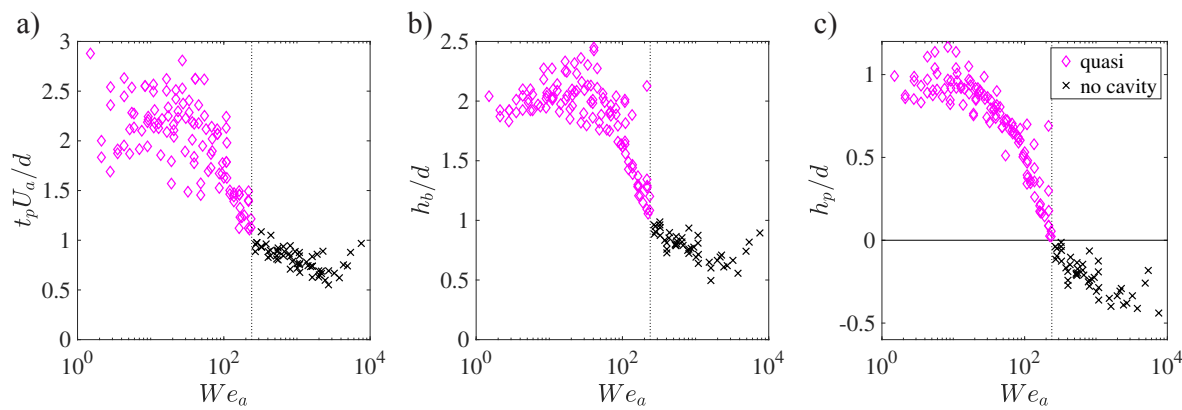

Figure 4 . a) The non-dimensional pinch-off time $t_{p}$, depth of the bottom of the sphere at pinch-off $h_{b}$ (inset) and b) the pinch-off depth $h_{p}$ scale with $W e_{a}$ for the quasi-static and no cavity regimes for $\theta=86.1^{\circ}$ and $101.0^{\circ}$. Figure $3 \mathrm{c}$ shows the same trends for $h_{b}$ and $h_{p}$ qualitatively except that here the surface seal data is not shown as transition occurs at a constant velocity and the data does not collapse with $W e_{a}$. The dotted line represents the splash formation line. Note that the Weber number and dimensionless pinch-off time are defined using the average sphere velocity from initial impact to full submergence $U_{a}\left(W e_{a}=\rho U_{a}^{2} d / \sigma\right)$. This accounts for the contiuned acceleration of the sphere when it has not reached the terminal velocity in water by the time it impacts (e.g., an $18 \mathrm{~mm}$ sphere falling $3 \mathrm{~mm}$ ). The legend in b) applies to all three plots.

(2016) that pop leaving holes in the crown. Partial surface seal occurs most commonly just above the critical velocity for cavity formation and at lower contact angles.

At the highest contact angle tested, when $\theta=141.1^{\circ}$ Duez et al. (2007) predict that cavities should form above a critical velocity of $U_{c r}=\frac{7}{270} \frac{\sigma}{\mu}(\pi-\theta)^{3}=0.58 \mathrm{~m} / \mathrm{s}$. This velocity gives $W e<240$ for all sphere diameters tested. Hence, the Duez cavity formation line lies below the splash formation line at all Bo tested and we would expect cavities to form at all impact velocities. This is indeed the case as shown in figure $2 \mathrm{~d}$. Comparing figure $2 \mathrm{~d}$ to the data obtained by Aristoff \& Bush (2009) for rough spheres with $\theta=120^{\circ}$ (shown in figure 2c) we see that the regime locations for the two coatings are very similar. Discrepancies in the cut off between quasi-static seal and shallow or deep seal are possibly due to the lower atmospheric pressure in Logan, Utah (elevation of $1382 \mathrm{~m}$ ) where our 


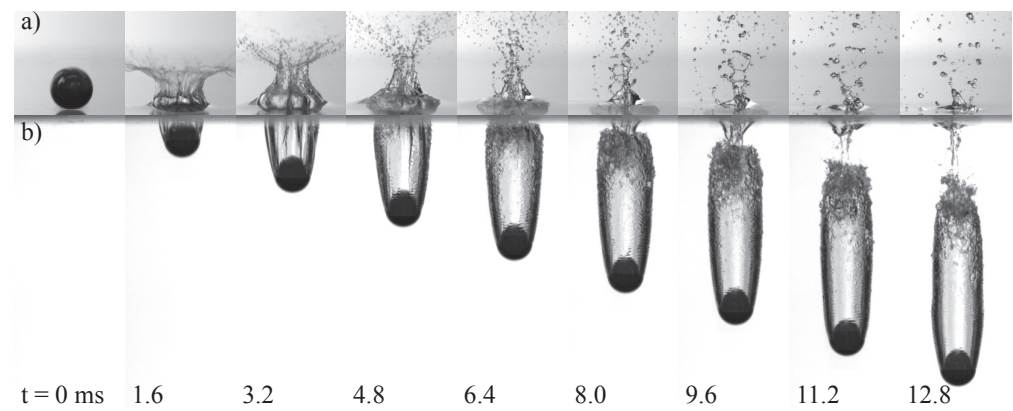

Figure 5. A sphere with $d=10 \mathrm{~mm}$ and $\theta=101.1^{\circ}$ impacts the pool surface at $U=6.26$ $\mathrm{m} / \mathrm{s}$ forming a surface seal cavity with the above and below water views shown in a) and b) respectively. The splash crown does not always provide a complete seal of the cavity during dome over. Sometimes it mostly seals, but allows some air continue to enter the cavity as shown by the conical air pocket near the surface and above the main portion of the cavity at $t=9.6 \mathrm{~ms}$. The conical portion of the cavity then collapses radially $(t=11.2-12.8 \mathrm{~ms})$. See supplementary movie 6 .

experiments were performed and Cambridge, Massachusetts (elevation of $40 \mathrm{~m}$ ) where the experiments of Aristoff \& Bush (2009) were performed, with the lower pressure leading to less air entrainment as shown by Gilbarg \& Anderson (1948), persisting for larger We. Discrepancies could also be due to the difference in roughness or the difference in wetting angle, or all three effects combined.

Spheres rebound off of the pool surface at the lowest $B o$ and $W e$ tested for all three contact angles. Figure 2 shows that transition from water entry to rebound occurs when $W e=1$ and $B o<1$. In this parameter space surface tension dominates over both inertial and gravitational forces and hence, neither the sphere's inertia nor its weight cause it to enter the water surface. Figure 2 also shows that rebound is slightly dependent on the sphere's contact angle, with higher contact angles leading to rebound at higher $B o$ and $W e$.

\section{A new scaling}

When defining dimensionless numbers it is always difficult to pick the appropriate length and velocity scales to describe the physics of the problem. Historically in water entry research, the sphere diameter or radius has been chosen as the length scale and the initial impact velocity for the velocity scale (Truscott et al. 2014). As the cavity collapse is likely to be a function of the cavity characteristics, it could be insightful to redefine the appropriate dimensionless numbers using cavity length and velocity scales. We define the cavity Weber number as $W e_{c}=\rho U_{c}^{2} d_{c} / \sigma$, the cavity Bond number as $B o_{c}=\rho g d_{c}^{2} / \sigma$ and the cavity Froude number as $F r_{c}=U_{c}^{2} / g d_{c}$, where $d_{c}$ is the cavity diameter defined in $\S 2$ and $U_{c}$ is the downward cavity velocity, which we set equal to the initial sphere impact velocity $U_{o}$ as they are approximately the same. Plotting a regime diagram with the cavity scaling for $\theta=141.1^{\circ}$, we see in figure 6 that $B o_{c}$ and $W e_{c}$ separate the cavity types for shallow, deep and surface seal (hollow symbols). Quasi-static seal cavities are not included because they are not cylindrical and they are specific to sphere entry as discussed more in the next paragraph.

Speirs et al. (2018) investigated the water entry of multi-droplet streams and jets and found that shallow, deep and surface seal cavities occur for liquid-liquid impact as well. In that paper we predicted the cavity seal types for both multi-droplet streams and jets on the same $B o-W e$ regime diagram using a scaling based on the cavity diameter and no 


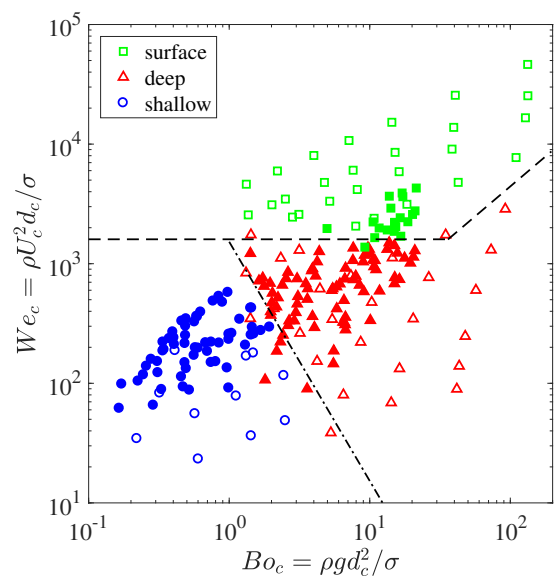

FIGURE 6 . The cavity regimes can be predicted for various impacting bodies using the cavity diameter $d_{c}$ and cavity velocity $U_{c}$ to define $B o_{c}$ and $W e_{c}$. The hollow symbols are for spheres and the solid symbols are for multi-droplet streams and jets. The dash-dotted line separating the shallow and deep seal regimes is found by equating the dimensionless pinch-off times for shallow and deep seal (figure 7). The dashed line dividing deep and surface seal is drawn by modifying the cutoffs found by Aristoff \& Bush (2009) and Birkhoff \& Isaacs (1951).

alteration of the impact velocity. We can collapse those data onto the $B o_{c}-W e_{c}$ regime diagram for spheres using $d_{c}$ and $U_{c}$ to define $B o_{c}$ and $W e_{c}$ as in figure 6 . The cavity velocity $U_{c}$ is set equal to one half the impacting stream velocity for simplicity, which is shown to be a good approximation for jets in Speirs et al. (2018). Using this scaling, figure 6 shows that the regimes for all three water entry types (spheres, liquid jets and liquid multi-droplet streams) can be predicted in the same $B o_{c}-W e_{c}$ parameter space. This scaling suggests that we can predict the pinch-off type of a cavity if we know its diameter and downward velocity, regardless of the type of impacting body used.

The cavity nondimensional pinch-off times, $t_{p} U_{c} / d_{c}$, of the sphere, multi-droplet stream and jet data can be predicted on the same plot for shallow and deep seal using $W e_{c}$ and $F r_{c}$, respectively. Figure 7 a shows that $t_{p} U_{c} / d_{c}$ scales with $W e_{c}^{1 / 2}$ for shallow seal and figure $7 \mathrm{~b}$ shows that $t_{p} U_{c} / d_{c}$ scales with $F r_{c}^{1 / 3}$ for deep seal. Equating these nondimensional pinch-off times and rearranging gives the cut-off between the shallow and deep seal regimes as shown by the dash-dotted line in figure $6\left(W e_{c}=1,525 B o_{c}^{-2}\right)$.

To predict the cut-off for surface seal we look at previous works. Aristoff \& Bush (2009) used an empirical fit of $W e=640$ to define the cut-off for surface seal at low $B o$ while at high $B o$ Birkhoff \& Isaacs (1951) predicted the cut-off to occur at $F r=1 / 12800\left(\rho / \rho_{a}\right)^{2}$, where $\rho_{a}$ is the air density. We can use these results to find the surface seal cut-off in terms of $W e_{c}$ and $F r_{c}$. At low $B o_{c}$, the cut-off is $W e_{c}=640\left(d_{c} / d\right)_{\text {mean }}$, where $\left(d_{c} / d\right)_{\text {mean }} \approx 2.5$ is the mean cavity to sphere diameter ratio for the deep and surface seal data just above and below the transition at low $B o_{c}$. This leads to a transition at $W e_{c}=1600$. At high $B o_{c}$, the cut-off is $F r_{c}=1 / 12800\left(\rho / \rho_{a}\right)^{2}\left(d / d_{c}\right)_{\text {mean }}$, where $\left(d / d_{c}\right)_{\text {mean }} \approx 1.6$ is the mean for the deep and surface seal data just above and below the transition at high $B o_{c}$. This leads to a transition at $F r_{c}=44$ or $W e_{c}=44 B o_{c}$. These transitional lines are shown in figure 6 with the dashed line and appropriately divide the deep and surface seal regimes. It is interesting to note that the shallow, deep and surface seal transition lines intersect at $B o_{c}=1$, indicating that when surface tension dominates over gravitational forces, shallow seal will always occur instead of deep. 

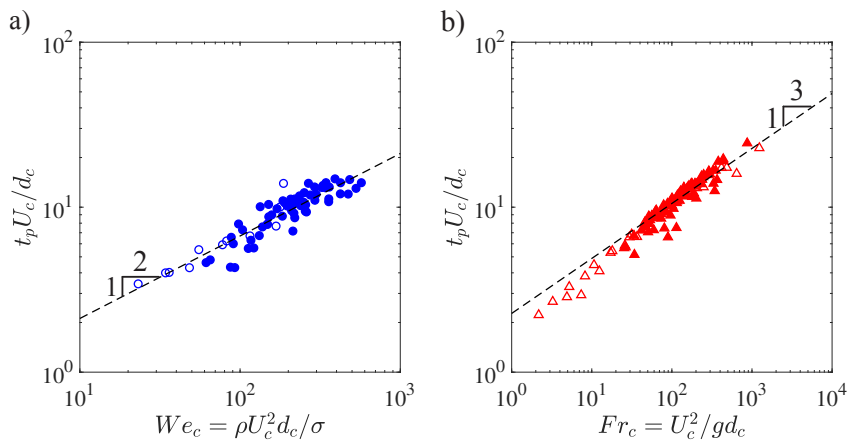

FiguRE 7. The nondimensional cavity pinch-off time scales with $W e_{c}$ and $F r_{c}$ for a) shallow and $\mathrm{b}$ ) deep seal for spheres with contact angle $\theta=141.1^{\circ}$ (hollow symbols) and multi-droplet streams and jets (solid symbols). The dashed lines are fits to the data with powers forced to $t_{p} U_{c} / d_{c}=0.67 W e_{c}^{1 / 2}$ for shallow seal and $t_{p} U_{c} / d_{c}=2.27 \mathrm{Fr}_{c}^{1 / 3}$ for deep seal.
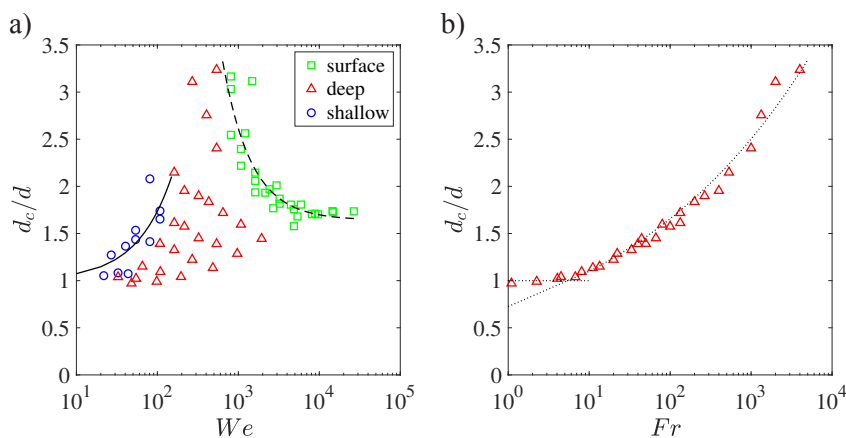

Figure 8 . The cavity diameter for the impact of spheres with $\theta=141.1^{\circ}$ scales with $W e$ for shallow and surface seal a) and $F r$ for deep seal b). The solid and dashed lines in a) are least square regressions with $d_{c} / d=0.0074 W e+1$ for shallow seal and $d_{c} / d=4952 W e^{-1.24}+1.64$ for surface seal. The curved dotted line in $\mathrm{b})$ is a fit for the deep seal data $\left(d_{c} / d=0.73 F r^{0.18}\right)$, but for $\mathrm{Fr}<7$ the cavity diameter approaches the sphere diameter, $d_{\text {cav }} / d=1$ marked by the horizontal dotted line.

Seeing the importance of the cavity diameter in calculating $B o_{c}$ and $W e_{c}$, we now scale $d_{c}$ for each pinch-off type. Plotting the nondimensional cavity diameter $d_{c} / d$ against $W e$ we see in figure $8 \mathrm{a}$ that the cavity diameter for the shallow seal data is a function of $W e$ and can be predicted with a linear fit of $d_{c} / d=0.0074 W e+1$, where the y-intercept is forced to equal one sphere diameter. We can also predict the cavity diameter for the surface seal data using $W e$ with the fit $d_{c} / d=4952 W e^{-1.24}+1.64$. As commonly seen, the deep seal data scales better with $F r$ as shown in figure $8 \mathrm{~b}$ and can be predicted by $d_{c} / d=0.73 F r^{0.18}$ for high $F r$, but below $F r \approx 7$ the ratio $d_{c} / d$ asymptotes to one.

\section{Conclusion}

Cavity formation is dependent on the formation and behavior of the splash crown. Three crown behaviors exist. 1) At low $W e$ a slow-moving, thick rim forms around the sphere, which allows air to entrain in the wake of the sphere forming small cavities. 2) At higher $W e$ the crown thins and gains velocity which allows it to adhere to the sphere, climb the surface, and meet at the apex to preventing cavity formation. 3) Once the critical velocity for cavity formation is reached the splash crown separates from the 
sphere forming the classical cavities discussed above and in previous works. The cutoffs between these behaviors are defined by splash formation and Duez cavity formation. For hydrophilic and slightly hydrophobic spheres, the inception of splash formation and adherence to the sphere decreases the cavity size, compared to higher contact angles, leading to quasi-static seal and small shallow seal cavities. When the contact angle is high enough $\left(\theta \gtrsim 140^{\circ}\right)$ or the sphere is rough (Zhao et al. 2014), cavities form at all impact velocities because the splash crown either does not form or it separates from the sphere. These cavity formation regimes are predicted by Aristoff \& Bush (2009). When cavity formation is not inhibited by the splash, the pinch-off type can be predicted by the cavity diameter and downward cavity velocity regardless of the type of impacting body (e.g., sphere, jet, or multi-droplet stream). This forms a more complete picture, linking the impact of solids and liquids on liquid pools.

\section{Acknowledgments}

N.B.S., T.T.T. and J.B. acknowledge funding from the Office of Naval Research, Navy Undersea Research Program (grant N0001414WX00811), monitored by Ms. Maria Medeiros. J.B. acknowledges funding from the Naval Undersea Warfare Center In-House Laboratory Independent Research program, monitored by Mr. Neil Dubois. N.B.S and T.T.T acknowledge funding from the Utah State University Research and Graduate Studies Development Grant Program.

\section{REFERENCES}

Aristoff, J. M. \& Bush, J. W. M. 2009 Water entry of small hydrophobic spheres. Journal of Fluid Mechanics 619, 45-78.

Aristoff, J. M., Truscott, T. T., Techet, A. H. \& Bush, J. W. M. 2010 The water entry of decelerating spheres. Physics of Fluids 22 (3).

Birkhoff, G. \& IsAacs, R. 1951 Transient cavities in air-water entry. Navord Report 1490. Tech. Rep.

Duez, C., Ybert, C., Clanet, C. \& Bocquet, L. 2007 Making a splash with water repellency. Nat Phys 3 (3), 180-183.

Gilbarg, D. \& Anderson, R. A. 1948 Influence of atmospheric pressure on the phenomena accompnaying the entry of spheres into water. J. Appl. Phys. 19, 127-139.

Hurd, R. C., Belden, J., Jandron, M. A., Fanning, D. T., Bower, A. F. \& Truscott, T. T. 2017 Water entry of deformable spheres. Journal of Fluid Mechanics 824, 912-930.

Mansoor, M. M., Marston, J. O., Vakarelski, I. U. \& Thoroddsen, S. T. 2014 Water entry without surface seal: extended cavity formation. Journal of Fluid Mechanics $\mathbf{7 4 3}$, 295-326.

Marston, J. O., Truscott, T. T., Speirs, N. B., Mansoor, M. M. \& Thoroddsen, S. T. 2016 Crown sealing and buckling instability during water entry of spheres. Journal of Fluid Mechanics 794, 506-529.

Speirs, N. B., Pan, Z., Belden, J. \& Truscott, T. T. 2018 The water entry of multi-droplet streams and jets. Journal of Fluid Mechanics 844, 1084-1111.

Truscott, T. T., Epps, B. P. \& Belden, J. 2014 Water entry of projectiles. Annual Review of Fluid Mechanics 46 (1), 355-378.

Truscott, T. T., Epps, B. P. \& Techet, A. H. 2012 Unsteady forces on spheres during free-surface water entry. Journal of Fluid Mechanics 704, 173-210.

Truscott, T. T. \& Techet, A. H. $2009 a$ A spin on cavity formation during water entry of hydrophobic and hydrophilic spheres. Physics of Fluids 21 (12), 121703.

Truscott, T. T. \& Techet, A. H. $2009 b$ Water entry of spinning spheres. Journal of Fluid Mechanics 625, 135-165.

Zhao, M.-H., Chen, X.-P. \& Wang, Q. 2014 Wetting failure of hydrophilic surfaces promoted by surface roughness. Scientific Reports 4, 5376 . 